\title{
versants
}

\section{Henri Roorda : le charme d'un suicide}

\author{
Yla VON DACH \\ Traductrice littéraire
}

\begin{abstract}
Les différentes possibilités qui s'offrent à la traduction en allemand du titre Mon suicide ouvrent non seulement sur une exploration de ce qui caractérise l'approche de Henri Roorda dans sa critique de la société, mais également sur une réflexion quant aux façons de nommer ou de ne pas nommer les choses et aux implications de la tendance actuelle à éviter "d'appeler les choses par leur nom", de peur de heurter les sensibilités.
\end{abstract}

Keywords : Henri Roorda, traduction, suicide, allemand, nuances de sens, franc-parler, langage voilé.

«Ce qui rend le mot infiniment précieux, c'est, plus que tout le reste, son pouvoir évocateur.»

«Si l'on m'obligeait à découvrir en moi une intention belliqueuse, je dirais qu'il me serait particulièrement agréable de déplaire aux fanatiques. »

Henri Roorda, «Le débourrage des crânes est-il possible? », I924 (9, 47-48).

Oui, je suis tombée sous le charme de Roorda en lisant son texte intitulé Mon suicide.

Il faut dire tout de suite peut-être que le titre initial de ce petit ouvrage, celui qui plaisait vraiment à l'auteur, était quelque peu différent : Le Pessimisme joyeux.

Et l'on sait le pourquoi de ce changement, puisque Roorda lui-même s'en explique - sous le nom de son double, Balthasar - dans les premières pages de son texte :

Après réflexion, je me dis que «Pessimisme joyeux » est une expression qui pourrait faire hésiter quelques acheteurs. Mon suicide sera un titre plus alléchant. Le public a un goût prononcé pour le mélodrame (Roorda $201 \mathrm{I}$ : 5I).

Ce constat, on s'en souviendra, précède l'annonce que, pour procurer un peu d'argent à ses créanciers, l'auteur songe à faire de son suicide un spectacle public, payant, bien sûr, dans le Grand Café de son ami Fritz, avec un prix des consommations triplé - idée à laquelle il renonce finalement parce que son "suicide pourrait laisser une tache ineffaçable sur le plancher de son honorable établissement » (Roorda 20II : 52). 
Et nous voilà pris dans le filet charmant de notre candidat au suicide, quitte à oublier la gravité de l'intention. Ah bon ?!, c'est juste le souci de la tache qui le fait renoncer à passer à l'acte? Quelle blague ! On pourrait croire que le projet se résumerait tout entier à cette image distordue qui est le propre de ce qui nous fait rire - mais il n'en est rien.

Tout en laissant vibrer, entre les lignes, son joyeux pessimisme, Roorda maintient le cap tout au long de l'écriture de ce dernier livre qu'il choisit définitivement d'intituler Mon suicide.

Est-ce que j'oserai l'avouer? J'en suis heureuse. Non pas de l'acte commis par l'auteur, cet homme si attachant, mais du titre maintenu.

Pour ma traduction, je n'ai pas hésité, en allemand, à choisir le mot "Selbstmord", qui, lui, est toutefois plus dur que le terme français, puisqu'il nomme de manière directe un aspect de cet acte : le "Mord", la mise à mort, le meurtre de soi-même.

Il y aurait eu d'autres possibilités : "Mein Suizid", par exemple. Ou même : "Mein Freitod". Le premier titre aurait bénéficié d'un petit voile adoucissant dû à l'emprunt du latin, le deuxième aurait impliqué - selon ma manière de le ressentir - à la fois un jugement moral à tendance approbatrice, mais un petit voile également, puisque l'affirmation de la liberté de l'acte jette une lumière quelque peu atténuante sur ce dernier, alors qu'il est très certainement précédé d'un tourment profond pour l'être qui s'apprête à le commettre.

J'ai donc choisi le terme qui m'a paru le plus direct, le plus cru, le plus honnête en quelque sorte - le plus dépourvu de quelque volonté qui soit pas de manipulation (embellir, voiler) ni de jugement (interdire, condamner, approuver), puisque simple constat brut, mathématique, si j'ose dire.

Il y a là quelque chose que je ressens comme une preuve de fidélité à Henri Roorda - et j'essayerai ici de suivre la trace de ce sentiment pour me l'expliquer à moi-même et à toutes celles et tous ceux qui, éventuellement, voudront m'accompagner dans cette petite exploration.

En effet, ce qui paraît tellement attachant dans le personnage d'Henri Roorda, tel qu'il nous apparaît dans les pages de Mon suicide, c'est sa sincérité désarmante servie par un regard impitoyablement lucide - imprégnée d'une bienveillance cependant, dont jamais, jamais il ne se départit. En plus, qu'il s'agisse de sa propre personne ou de personnages ou faits de société de son temps, on est souvent tenté d'attribuer à Roorda quelque faculté prophétique, tant les vues et les considérations de cet observateur aussi partial qu'impartial semblent préfigurer l'évolution dont nous avons été témoins au cours de toutes les décennies qui ont suivi sa mort.

De lui-même, il dit à un moment donné : « Mais je ne peux pas me juger avec une grande sévérité, car j'ai toujours eu d'excellentes intentions » (Roorda 20II : 60). Et aussi : « Mon intelligence de luxe ne m'a jamais aidé à 
devenir plus fort ; le délicat que je suis était fait pour dépenser aristocratiquement l'argent gagné par les autres » (Roorda 20II : 6I-62).

Je fonds, en lisant ces lignes!

Car ce qui, chez certains, pourrait faire penser à une excuse bon marché et méprisable (pour ce qui est de la première citation) ou au comportement condamnable d'un de ces "hommes blancs" auxquels on fait volontiers le procès (souvent justifié, souvent bon marché) aujourd'hui, m’apparaît chez Roorda comme l'incarnation d'un paradoxe, en quelque sorte. Tout en nommant les choses sans tenter de les couvrir d'un voile atténuant qui lui permettrait de sauver la face ou de s'accorder quelque excuse par derrière, Roorda suscite en nous non pas un jugement un peu sévère, mais une douce réaction d'indulgence ; une indulgence du cœur plutôt que de la raison, car là où la raison serait tout de même encline à désapprouver, le cœur est sensible à la dimension tragique qui, mine de rien, transparaît dans ces constats.

Et cette dimension tragique, nous la connaissons tous, intimement, à des degrés divers et à des intensités différentes selon le cas, à travers nos propres conflits intérieurs, nos propres contradictions inextricables.

Comment cela se fait-il ? Comment Roorda arrive-t-il à nous faire regarder ainsi les choses en face sans vouloir nous voiler la face ni tomber dans le rejet d'un jugement moral?

Il y a son humour, bien sûr, qui imprègne même ses phrases pas aussi ouvertement humoristiques que celle évoquée au début. Mais il y a autre chose aussi. Il se peut que ce soit justement cette façon de se livrer sans fard, qui, plus que toute autre manière de parler, fasse résonner en nous une couche de notre être qui se connaît également sans fard, même si la plupart du temps, nous n'oserions pas nous exposer ainsi aux yeux de tous, de peur d'être condamnés ou mal compris.

Roorda, décidé comme il était de passer à l'acte, avait sans doute baissé la garde, d'une certaine manière.

Mais il a toujours eu dans ses textes, et non seulement dans Mon suicide, ce même regard direct, cette même audace de nommer les choses sans ambages. Et de l'humour merveilleux dont il était doté, il n'usait jamais pour simplement détourner du sérieux de ce qu'il dénonçait, ni pour introduire quelque hargne dans ses propos, comme cela peut se faire aussi (notre ère de communications déchaînées nous en montre des exemples en veux-tu en voilà).

Pour ce qui est de son regard sur la société, qui oserait encore parler si crûment et si simplement aujourd'hui de ce qui, au fond, est pourtant toujours aussi vrai un bon siècle plus tard ; qui serait capable de dire tout cela de cette manière à la fois si directe, si placide et si implacable?

Largent fait le bonheur. Pendant la grande guerre de I9I4, des hommes riches ont sacrifié généreusement leurs enfants sur l'autel de la Patrie. Mais, 
plus tard, quand la Patrie a eu besoin d'argent, ces hommes vertueux ont mis leur fortune en lieu sûr. Leur conscience ne leur a pas commandé d'aller jusqu'au sacrifice suprême (Roorda 2011 : 57).

Si nos fonds de commerce sont restés les mêmes, notre façon de nommer les choses, elle, a changé.

Et j'avoue que, très souvent, j'éprouve un malaise par rapport aux usages actuels où l'on parlera par exemple du "pronostic vital engagé" pour une personne blessée dans un accident ou par une agression - en évitant soigneusement tout ce qui pourrait évoquer explicitement la mort dans notre conscience.

On ne parle plus non plus de "femme de ménage", d'“homme de ménage", si je ne me trompe. Ce sont des "techniciennes/techniciens d'entretien", de préférence. Et que penser du terme "agents de propreté urbaine" pour désigner ceux qui, autrefois, devaient s'appeler "éboueurs" et "balayeurs de rue" ? J'entends bien que, dans ces dénominations anciennes, on peut également voir l'expression d'un état d'esprit très peu sympathique. On y perçoit la résonance d'un élément de mépris, collectif et individuel, que l'on souhaite à juste titre effacer et que l'on espère peut-être éliminer en changeant les mots. Pendant la période actuelle marquée par la pandémie du Covid-ı 9 et le confinement que certains ont vécu, nous avons pu voir quel rôle fondamental jouent justement les personnes évoquées à l'instant, aux métiers peu considérés : les infirmières et infirmiers en premier lieu, mais également les caissières, les éboueurs, par exemple, et j'en oublie, qui assurent ainsi dans l'anonymat des tâches finalement essentielles pour le collectif que nous sommes. Et pourtant, je ne peux nier un certain malaise quand je pense à cette nouvelle façon de nommer ou ne pas nommer les choses.

Qu'est-ce qui se passe en nous, avec nous, quand nous entendons dire que "le pronostic vital de quelqu'un est engagé"? Qu'est-ce qui se passe si des mots qui peuvent faire peur sont évités?

Le fait de nommer la femme de ménage "technicienne d'entretien" aurait-il vraiment le pouvoir de changer la considération que nous avons pour la personne et le travail qu'elle effectue ? Est-ce que les esprits ont changé au point de vraiment tenir ces personnes en plus grande estime, et surtout de leur accorder également un salaire un peu plus conforme à l'importance qu'on leur a reconnue à un instant critique de notre vie commune?

Voyez-vous : il me semble que ce n'est pas tant une question de mots et de terminologie, qui devraient changer, si l'on a vraiment l'intention de changer les choses. Ce qui nous fait défaut, et ce dont Henri Roorda nous gratifie avec tant de générosité toujours, sans pour autant nous épargner une parole sans détours, c'est cette bienveillance profonde qui sous-tendait tout son être. 
Est-ce que de changer les mots suffira pour nous faire accéder à cette qualité de cœur?

On peut en douter, même si l'espoir est toujours permis.

C'est certainement une bonne chose que de prendre conscience des implications sous-jacentes de certaines manières de parler; on ne peut donc que saluer cette évolution. Cependant, il ne faudrait pas oublier que par des mots voilés, nous pouvons également, très facilement, nous voiler la face.

En faisant disparaître de notre conscience tout ce qui, de près ou de loin, pouvait encore nous rappeler la saleté et les mauvaises odeurs auxquelles se voient exposés les éboueurs chargés du travail si ingrat d'enlever nos déchets; en éliminant toute allusion à ce qui, dans les mots, peut nous rappeler des faits réels peu agréables, voire angoissants, en nous réfugiant dans un langage aux concepts tout à fait aseptisés, il se peut bien que nous perdions également le contact avec notre ressenti immédiat, qui est sans doute l'une des conditions préalables à une véritable empathie. Curieusement, la volonté affichée de gratifier certaines personnes d'une plus grande considération ou de faire preuve de plus de délicatesse dans la façon de nommer les choses dont se réclame apparemment cette drôle de terminologie pourrait même aboutir à un effet contraire ! Au lieu de nous rendre plus empathiques, plus bienveillants, plus respectueux, il se peut qu'elle nous rende en quelque sorte plus abrutis! Je m'explique : comment des mots au pouvoir d'évocation si aseptisé peuvent-ils toucher cette dimension sensible qui seule, associée à la compréhension intellectuelle, fait de nous des êtres entiers, marchant même intérieurement sur nos deux pieds ? Entre parenthèses, et en extrapolant peut-être outre mesure, on peut même se demander si ce genre de langage n'est pas susceptible d'ouvrir la voie à ces beaux parleurs au langage creux que sont toujours et à nouveau les populistes, voire les fanatiques, dont Henri Roorda a d'ailleurs toujours très finement perçu les signes et les relents... Le monde, tel qu'il se présente à nous actuellement, devrait en tout cas nous inciter à veiller au grain et à ne pas considérer tout cela comme de la pure subtilité.

Mais revenons encore une fois à Henri Roorda, écoutons une fois de plus le timbre incomparable de cet équilibre qu'il a su trouver, et garder, dans ses reproches adressés à lui-même et aux autres, entre franche dénonciation et calme impassible, entre culpabilité et désespoir infinis allant de pair avec une douceur à mon sens toute aussi infinie :

Je vais bientôt me tuer. Je ne mérite pas ce châtiment. Je suis sûr d'avoir eu moins de vilaines pensées que la plupart de ces bons citoyens qui réussissent et qui ne songeront jamais à se suicider. Les beaux vers que je me récitais mettaient de la pureté dans mon esprit. Ils m'ont procuré chaque jour une minute d'émotion. Ah ! je voudrais bien rester sur la terre!

Lorsqu'on est totalement dépourvu de méchanceté, on peut faire, quand 
même, énormément de mal. Je voudrais demander pardon à quelqu'un, mais les mots que je devrais dire n'existent pas (Roorda $201 \mathrm{I}$ : 77-78).

Oui, il n'était pas parfait, Henri Roorda, comme personne de nous n'est parfait.

S'est-il pardonné ? Il s'est suicidé tout en se pardonnant peut-être.

Nous, on ne peut que lui pardonner, me semble-t-il. Et garder en mémoire, comme un cadeau précieux, ce "corpus" de textes vibrant de toutes ces incompatibilités de beautés, de désirs, de culpabilités, d'incapacités et de fautes assumées - où quelque chose semble dominer malgré tout, entre les lignes tout comme dans le drame de sa vie et de sa mort : j'oserai le nommer "amour".

\section{Bibliographie}

Roorda, Henri, Le débourrage des crânes est-il possible?, Lausanne, Vaney-Burnier, I924.

—. Le Rire et les rieurs suivi de Mon suicide, Paris, Mille et Une Nuits, 20 II. 